\title{
Extension of Dirac's Substraction Formalism to Particles of Finite Size
}

\author{
J. Petzold \\ Fachbereich Physik der Universität Marburg
}

Z. Naturforsch. 35a, 675-678 (1980); received May 19, 1980

Extension of Dirac's Subtraction Formalism to Particles of Finite Size

Dirac's subtraction formalism is extended to radiating particles of finite size. Irrespective of the charge distribution the equation of motion contains run-away solutions. Hence the radiation reaction is not well described by $F_{-}^{\mu \nu}$.

\section{Einleitung und Zusammenfassung}

In der klassischen Theorie der Strahlungsreaktionen* ist der Ausgangspunkt für die Beschreibung der Rückwirkung des durch Beschleunigungen erzeugten Strahlungsfeldes auf ein Teilchen die Lorentzsche Kraftdichte**

$$
k^{\mu}\left(x^{\varrho}\right)=s_{\nu}\left(x^{\varrho}\right) F_{\text {ret. }}^{v \mu}\left(x^{\varrho}\right),
$$

wobei $s_{\nu}$ die elektrische Viererstromdichte und $F_{\text {ret. }}^{v \mu}$ das retardierte Eigenfeld des Teilchens ist.

Unter Einbeziehung der avancierten Lösungen $F_{\text {av. }}^{\nu \mu}$ der Maxwell-Gleichungen führt man die Felder

$$
\begin{aligned}
F_{+}^{\nu \mu} & =\frac{1}{2}\left\{F_{\text {ret. }}^{v \mu}+F_{\text {av }}^{v \mu}\right\} ; \\
F_{-}^{\nu \mu} & =\frac{1}{2}\left\{F_{\text {ret. }}^{v \mu}-F_{\text {av. }}^{v \mu}\right\} ; \\
F_{\text {ret. }}^{v \mu} & =F_{+}^{v \mu}+F_{-}^{v \mu}
\end{aligned}
$$

und

$$
k_{+}^{\mu}=s_{\nu} F_{+}^{\nu \mu} ; \quad k_{-}^{\mu}=s_{\nu} F_{-}^{v \mu}
$$

ein. $F_{+}^{v \mu}$ ist symmetrisch unter Zeitspiegelungen, enthält die Coulomb-Energie und liefert keinen Beitrag zum Strahlungsfeld, während die Antisymmetrie von $F_{-}^{\nu \mu}$ hinreichend für die Beschreibung der Energie-Dissipation ist. $F_{-}^{v \mu}$ erfüllt die freien Maxwell-Gleichungen, wie man es vom Strahlungsfeld erwartet. Die Reaktionskraft sollte vollkommen in $K_{-}^{\mu}=\int k_{-}^{\mu} \mathrm{d}^{3} x$ stecken.

Es soll in dieser Arbeit gezeigt werden, daß sich $K_{+}^{\mu}=\int k_{+}^{\mu} \mathrm{d}^{3} x$ als zeitliche Ableitung einer Größe $P_{+}^{\mu}$ darstellen läßt $\left(K_{+}^{\mu}=-\mathrm{d} P_{+}^{\mu} / \mathrm{d} x^{0}\right)$, die nur vom

Reprint requests to J. Petzold, Fachbereich Physik der Universität, Mainzer Gasse 33, D-3550 Marburg.

* Eine zusammenfassende Darstellung findet man bei Th. Erber: The Classical Theories of Radiation Reaction [1].

** $\left\{x^{v}\right\}=\left\{x^{0}, x^{1}, x^{2}, x^{3}\right\}=\left\{x^{0}, x^{n}\right\} ;$ Sig $\left\{g^{v \mu}\right\}=\{+---\} ;\left.f\right|_{v}\left(x^{\varrho}\right)=\partial f\left(x^{\varrho}\right) / \partial x^{\nu}$.
Teilchenzustand in einem kleinen Zeitintervall von der Größenordnung des Teilchendurchmessers in der Umgebung des betrachteten Zeitpunktes $x^{0}$ abhängt. Diese Abhängigkeit von einem Bahnsegment impliziert eine gewisse Nicht-Lokalität in dem Ausdruck von $P_{+}^{\mu}(\operatorname{vgl}$. $\S I I)$, hervorgerufen durch die Wechselwirkung des Teilchens mit sich selbst.

Falls das Teilchen stabil bleiben soll, müssen mechanische Spannungen die abstoßenden CoulombKräfte kompensieren, beide von gleicher räumlicher Ausdehnung. Obwohl jene Spannungen im Prinzip unbekannt sind, können sie doch nach den Gesetzen der Relativitätstheorie Wirkungen nur mit Schallgeschwindigkeit, d.h. Unter-Lichtgeschwindigkeit weitergeben. Einen absolut starren Körper gibt es nämlich nicht [2]. Es treten also auch hier wie bei den elektromagnetischen Kräften Retardierungseffekte auf, die sich wie dort in einer Art nichtlokaler Wechselwirkung zeigen müssen. Wenn die mechanischen Kräfte kein Gedächtnis besitzen, keine Hysteresis-Effekte auftreten, dann sollten sich die mechanischen Kräfte in einem mechanischen Impuls $P_{\text {mech. }}^{\mu}$ manifestieren, der eine ähnliche Struktur wie $P_{+}^{\mu}$ hat. Dann kann man umgekehrt auch $P_{+}^{\mu}$ als einen Trägheitsterm ansehen.

Man mag nun die Hoffnung hegen, daß sich die elektromagnetischen Nicht-Lokalitäten in $P_{+}^{\mu}$ und die entsprechenden mechanischen von $P_{\text {mech. gegen- }}^{\mu}$ seitig kompensieren, so daß man beide zu einem lokalen Gesamtimpuls

$$
P^{\mu}=P_{+}^{\mu}+P_{\text {mech. }}^{\mu}=m u^{\mu}
$$

zusammenfassen kann. Dabei wäre dann $m$ die Gesamtmasse des Teilchens.

Da weder $P_{+}^{\mu}$ noch $P_{\text {mech. }}^{\mu}$ Beiträge zur Reaktionskraft liefern, könnte die Zusammenfassung zumindest in guter Näherung sinnvoll sein. Es wären dann 
gewisse Ansätze in der Literatur gerechtfertigt. Also sollte (vgl. z.B. [1], S. 351)

$$
\frac{\mathrm{d}}{\mathrm{d} x^{0}} P^{\mu}=\frac{\mathrm{d}}{\mathrm{d} x^{0}} m u^{\mu}=K_{-}^{\mu}+K_{\mathrm{äu}}^{\mu} .
$$

eine sinnvolle Bewegungsgleichung sein, insbesondere da die für Punktteilchen divergente Coulomb-
Selbstenergie durch Absorption in $P^{\mu}$ formal eleminiert wurde. Eine Analyse von $K_{-}^{\mu}$ zeigt aber, daß es zu Selbstbeschleunigungslösungen Anlaß gibt, unabhängig von der Größe der Gesamtmasse. Die Zerlegung des elektromagnetischen Feldes nach (I, 2a) scheint also unphysikalisch zu sein, womit eine in der Literatur ([1], S. $350 \mathrm{ff}$.) gehegte Hoffnung sich als unbegründbar erweist.

\section{Darstellung von $K_{+}^{\mu}$ als zeitliches Differential}

Um die erwähnte Darstellung von $K_{+}^{\mu}$ zu finden, führen wir zunächst die Vektorpotentiale ein

$$
A_{\text {ret. }}^{\mu}\left(x^{\varrho}\right)=\int D_{\substack{\text { ret. } \\ \text { av. }}}\left(x^{\varrho}-\tilde{x}^{\varrho}\right) s^{\mu}\left(\tilde{x}^{\varrho}\right) \mathrm{d}^{4} \tilde{x}=\frac{1}{4 \pi} \int \frac{s^{\mu}\left(\tilde{x}^{r}, x^{0} \mp|\boldsymbol{x}-\tilde{\boldsymbol{x}}|\right)}{|\boldsymbol{x}-\tilde{\boldsymbol{x}}|} \mathrm{d}^{3} \tilde{x} .
$$

Wegen des Ladungserhaltungssatzes $s^{\mu}{ }_{\mid \mu}=0$ können wir $K_{+}^{\mu}$ in die Form

$$
K_{+}^{\mu}=\int s_{v} F^{v \mu} \mathrm{d}^{3} x=\int s_{v} A_{+}^{v \mid \mu} \mathrm{d}^{3} x-\int s_{v} A_{+}^{\mu \mid v} \mathrm{~d}^{3} x=\int s_{\nu} A_{+}^{v \mid \mu} \mathrm{d}^{3} x-\frac{\mathrm{d}}{\mathrm{d} x^{0}} \int s_{0} A_{+}^{\mu} \mathrm{d}^{3} x
$$

bringen. Für das erste Integral zeigt man unschwer

$$
\begin{aligned}
\int s_{\nu} A_{+}^{v \mid \mu} \mathrm{d}^{3} x= & \frac{1}{2} \int\left(s_{v} A_{+}^{v \mid \mu}-s_{\nu}{ }^{\mu} A_{+}^{\nu}\right) \mathrm{d}^{3} x+g^{\mu 0} \frac{\mathrm{d}}{\mathrm{d} x^{0}} \frac{1}{2} \int s_{\nu} A_{+}^{\nu} \mathrm{d}^{3} x=-\frac{\mathrm{d}}{\mathrm{d} x^{0}} \frac{1}{16 \pi} \int \mathrm{d}^{3} x \mathrm{~d}^{3} \tilde{x} \int_{0}^{1} \mathrm{~d} \lambda \\
& \cdot\left[s_{\nu}{ }^{\prime} \mu\left(x^{r}, x^{0}-(1-\lambda)|\boldsymbol{x}-\tilde{\boldsymbol{x}}|\right) s^{\nu}\left(\tilde{x}^{r}, x^{0}+\lambda|\boldsymbol{x}-\tilde{\boldsymbol{x}}|\right)-s_{\nu}\left(x^{r}, x^{0}-(1-\lambda)|\boldsymbol{x}-\tilde{\boldsymbol{x}}|\right)\right. \\
& \left.\cdot s^{v \mid \mu}\left(\tilde{x}^{r}, x^{0}+\lambda|\boldsymbol{x}-\tilde{\boldsymbol{x}}|\right)\right]+g^{\mu 0} \frac{\mathrm{d}}{\mathrm{d} x^{0}} \frac{1}{2} \int s_{\nu} A_{+}^{v} \mathrm{~d}^{3} x \equiv-\frac{\mathrm{d}}{\mathrm{d} x^{0}} p_{+}^{\mu} .
\end{aligned}
$$

Dabei überlegt man sich leicht, daß in den Integralen nur Raum-Zeit-Gebiete eine Rolle spielen, die von der Größenordnung des Teilchendurchmessers und derjenigen Zeiten sind, die das Licht zum Überstreifen des Teilchens benötigt (wobei diese sich nur mit Unterlichtgeschwindigkeit fortbewegen können). Also läßt sich $K_{+}^{\mu}$ als zeitliches Differential der Größe

$$
-P_{+}^{\mu}=-p_{+}^{\mu}-\int s_{v} A_{+}^{v} \mathrm{~d}^{3} x
$$

schreiben. Diese hängt nicht von der gesamten Teilchenbahn bzw. Vorgeschichte der Partikel ab, sondern nur von einem Bahnsegment von der Größenordnung des Teilchendurchmessers in der Umgebung der betrachteten Hyperfläche $x^{0}=$ const.

Äquivalent dazu kann man sagen, daß das ,,Wirkungsintegral"

$$
\int_{x_{1}{ }^{0}}^{x_{2}{ }^{0}} K_{+}^{\mu} \mathrm{d} x^{0}=-P_{+}^{\mu}\left(x_{2}^{0}\right)+P_{+}^{\mu}\left(x_{1}^{0}\right)
$$

nur von den Systemeigenschaften in der mikroskopischen Umgebung der Randpunkte $x_{i}^{0}$ abhängt.
Durch die Wegunabhängigkeit von $\int K_{+}^{\mu} \mathrm{d} x^{0}$ wird eine Art Zustandsgröße definiert. Hingegen ist die Rückwirkung $\int_{x_{1}{ }^{0}}^{x_{2}{ }^{0}} K_{-}^{\mu} \mathrm{d} x^{0}$ nicht unabhängig vom Weg des Teilchens zwischen den Randpunkten, vielmehr geht die ganze makroskopische Teilchentrajektorie zwischen $x_{1}^{0}$ und $x_{2}^{0}$ ein, so wie man es für einen Abstrahlungsterm erwartet; denn die abgestrahlten elektromagnetischen Felder sind bekanntlich von den jeweiligen Beschleunigungen abhängig.

\section{Analyse von $K_{-}^{\mu}$ in der linearen Näherung}

Das Strahlungsfeld $F_{-}^{v \mu}$, das den freien MawxellGleichungen genügt, stellt man bequem durch die Vektorpotentiale $A_{-\mathrm{C}}^{\mu}$ in der Coulomb-Eichung dar; denn für diese gilt hier $A_{-C}^{0} \equiv 0 ; A_{-C / n}^{n}=0$, so da $B$ man nur zwei unabhängige Felder zu berücksichtigen braucht. Explizit lautet

$$
\begin{aligned}
& A_{-C}^{n}\left(x^{\varrho}\right) \\
& \quad=\frac{1}{2} \int D_{\operatorname{tr} .}^{n m}\left(x^{\varrho}-\tilde{x}^{\varrho}\right) s_{m}\left(\tilde{x}^{\varrho}\right) \mathrm{d}^{4} \tilde{x}
\end{aligned}
$$


mit

$$
\begin{aligned}
D_{\mathrm{tr} .}^{n m}\left(x^{\varrho}\right)= & \frac{i}{(2 \pi)^{3}} \int \frac{\mathrm{d}^{3} k}{2 k^{0}}\left\{\mathrm{e}^{-i k_{\varrho} x^{\varrho}}-e^{i k_{\varrho} x^{\varrho}}\right\} \\
& \cdot\left\{g^{n m}+\frac{k^{n}}{k^{0}} \frac{k^{m}}{k^{0}}\right\}
\end{aligned}
$$

als transversaler Ausbreitungsfunktion des Lichtes. Ähnlich wie in § II erhält man

$$
\begin{aligned}
K_{-}^{\mu}\left(x^{0}\right)= & \int s_{n} A_{-C}^{n \mid \mu} \mathrm{d}^{3} x \\
& -\left(1-g^{\mu 0}\right) \frac{\mathrm{d}}{\mathrm{d} x^{0}} \int s_{0} A_{-C}^{\mu} \mathrm{d}^{3} x
\end{aligned}
$$

$$
\begin{aligned}
K_{-}^{m}\left(x^{0}\right) & \approx-\frac{\mathrm{d}}{\mathrm{d} x^{0}} \int s_{0} A_{-C}^{m} \mathrm{~d}^{3} x \\
& =-\frac{\mathrm{d}}{\mathrm{d} x^{0}} \int \varrho\left(x^{r}-\xi\left(x^{0}\right)\right) \frac{1}{2} D_{\mathrm{tr} .}^{m n}\left(x^{0}-\tilde{x}^{0}, x^{s}-\tilde{x}^{s}\right) \varrho\left(\tilde{x}^{r}-\xi\left(\tilde{x}^{0}\right)\right) v_{n}\left(\tilde{x}^{0}\right) \mathrm{d}^{3} x \mathrm{~d}^{4} \tilde{x} \\
& =-\frac{\mathrm{d}}{\mathrm{d} x^{0}} \int \varrho\left(x^{r}\right) \frac{1}{2} D_{\mathrm{tr} .}^{m n}\left(x^{0}-\tilde{x}^{0}, x^{s}-\tilde{x}^{s}+\xi\left(x^{0}\right)-\xi\left(\tilde{x}^{0}\right)\right) \varrho\left(\tilde{x}^{r}\right) v_{n}\left(\tilde{x}^{0}\right) \mathrm{d}^{3} x \mathrm{~d}^{4} \tilde{x}
\end{aligned}
$$

Das Integrationsgebiet ist von gleicher Art wie in $\S$ II beschrieben. Wir können in der betrachteten Näherung konsequenterweise die Abhängigkeit von

$$
\xi\left(x^{0}\right)-\xi\left(\tilde{x}^{0}\right)=\int_{\tilde{x}^{0}}^{x^{0}} v^{s}(\tau) \mathrm{d} \tau
$$

vernachlässigen und erhalten

$$
K_{-}^{m}\left(x^{0}\right) \approx-\frac{\mathrm{d}}{\mathrm{d} x^{0}} \int \varrho\left(x^{r}\right) \frac{1}{2} D_{\mathrm{tr} .}^{m n}\left(\tilde{x}^{0}, x^{s}-\tilde{x}^{s}\right) \varrho\left(\tilde{x}^{r}\right) \mathrm{d}^{3} x \mathrm{~d}^{3} \tilde{x} v_{n}\left(x^{0}-\tilde{x}^{0}\right) \mathrm{d} \tilde{x}^{0} .
$$

Weitere Vereinfachungen bringt die Annahme rotationssymmetrischer Ladungsdichten $\varrho=\varrho(r)$, denn mit (III, 2) erhält man

$$
\frac{1}{2} \int \varrho(r) D_{\mathrm{tr} .}^{m n}\left(\tilde{x}^{0}, x^{s}-\tilde{x}^{s}\right) \varrho(\tilde{r}) \mathrm{d}^{3} x \mathrm{~d}^{3} \tilde{x}=\frac{1}{3} g^{m n}\left(1 / \tilde{x}^{0}\right) M\left(\tilde{x}^{0}\right),
$$

wobei zur Abkürzung

$$
M\left(\tilde{x}^{0}\right)=(1 / 4 \pi) \int \varrho(r)\left\{\delta\left(x^{0}+|\boldsymbol{x}-\tilde{\boldsymbol{x}}|\right)+\delta\left(x^{0}-|\boldsymbol{x}-\tilde{\boldsymbol{x}}|\right)\right\} \varrho(\tilde{r}) \mathrm{d}^{3} x \mathrm{~d}^{3} \tilde{x}
$$

gesetzt wurde. Dann nimmt schließlich die Reaktionskraft die Form an:

$$
K_{-}^{m}\left(x^{0}\right) \approx-\frac{\mathrm{d}}{\mathrm{d} x^{0}} \frac{1}{3} \int M\left(\tilde{x}^{0}\right) \frac{1}{\tilde{x}^{0}} v^{m}\left(x^{0}-\tilde{x}^{0}\right) \mathrm{d} \tilde{x}^{0} .
$$

Für punktförmige Teilchen geht (III,6) unter Beachtung von (III, 5) in den bekannten Schott-Term

$$
K_{-}^{m} \approx \frac{2}{3} \frac{e^{2}}{4 \pi} \ddot{v}\left(x^{0}\right)
$$

über, der zu den Selbstbeschleunigungslösungen Anla $B$ gibt. Doch solche Lösungen treten auch dann auf, wenn man (III,6) in die Bewegungsgleichung für freie Teilchen (nach $(I, 3)$ ) einsetzt, ohne den
Übergang zu Punktteilchen zu machen (wobei entsprechend den Ausführungen des §I der nichtlokale Term $K_{+}^{\mu}$ und die mechanischen Anteile zusammengefaßt und in $m \dot{v}$ reduziert dargestellt werden):

$$
m \dot{v}\left(x^{0}\right)+\frac{1}{3} \int M\left(\tilde{x}^{0}\right) \frac{1}{\tilde{x}^{0}} \dot{v}\left(x^{0}-\tilde{x}^{0}\right) \mathrm{d} \tilde{x}^{0}=0 .
$$


Diese Gleichung kann man allgemein, wegen des Auftretens des Faltungsintegrals bezüglich der Zeit, mit Hilfe der Laplace-Transformation zu lösen versuchen. Da wir aber nur daran interessiert sind, das Auftreten von Selbstbeschleunigungslösungen nachzuweisen, gehen wir in (III, 7) mit dem Ansatz

$$
\dot{v}\left(x^{0}\right)=e^{\gamma x^{0}} \text { mit } \quad \gamma>0
$$

ein und erhalten unter Beachtung von $M\left(\tilde{x}^{0}\right)=$ $M\left(-\tilde{x}^{0}\right)$ für $\gamma$ die Gleichung

$$
m=\frac{1}{6} \int M\left(\tilde{x}^{0}\right) \frac{\operatorname{Sinh} \gamma \tilde{x}^{0}}{\tilde{x}^{0}} \mathrm{~d} \tilde{x}^{0} .
$$

Für definite Ladungsdichten $\varrho \geqq 0$ ist nach (III, 5 ) $M\left(\tilde{x}^{0}\right) \geqq 0$ und daher ist für $\gamma>0$ die rechte Seite von (III, 8) positiv. Sie verschwindet für $\gamma=0$ und wächst wegen

$$
\frac{1}{\tilde{x}^{0}} \operatorname{Sinh} \gamma x^{0} \geqq \gamma
$$

stärker als linear in $\gamma$ an. Daher gibt es immer ein $\gamma>0$, das für eine beliebige Gesamtmasse $m$ (III, 8) befriedigt. Da kein Übergang zum Punktteilchen gemacht wurde, ist die Coulomb-Energie endlich. Es treten also selbst dann Selbstbeschleunigungen auf, wenn die Gesamtmasse groß gegen die elektromagnetische Selbstenergie ist.

[1] Th. Erber, Fortschr. d Phys. 9, 343 (1961).

[2] M. v. Laue, Z. Phys. 12, 85 (1911).
Dieses Ergebnis scheint dem Herglotz-Wildermuth-Theorem zu widersprechen, nach dem Selbstbeschleunigungen dann auftreten, wenn die Gesamtmasse kleiner als die elektromagnetische ist ([1], S. $377 \mathrm{ff}$.). Das ist nicht der Fall, denn dort werden über die mechanischen Eigenschaften des Systems andere Annahmen als bei uns gemacht: sie werden allein durch einen lokalen Term

$$
\frac{\mathrm{d}}{\mathrm{d} x^{0}} m_{\text {mech. }} u^{\mu}
$$

dargestellt, was aber nach den Ausführungen von $\S$ I recht unphysikalisch ist.

Wenn man also die Bewegungsgleichung für ein geladenes, strahlendes Teilchen nach Dirac reduziert, treten unter ganz allgemeinen Bedingungen Selbstbeschleunigungslösungen auf. Diese sind nicht unbedingt eine Folge des Überganges zum punktförmigen Teilchen oder einer ungeschickten Wahl der Gesamtmasse. Führt man diese Reduzierung nicht durch, sondern behält die retardierten Felder konsequent bei, so kann man, ausgehend von der Lorentzschen Kraftdichte (I,1) zu einer IntegralDifferentialgleichung für die Teilchenbewegung kommen, die die oben erwähnten Mängel nicht enthält, sondern das Herglotz-Wildermuth erfüllt [3]

[3] D. J. Kaup, Phys. Rev. 152, 1130 (1966). 